\title{
A AMAMENTAÇÃO COMO MÉTODO NÃO FARMACOLÓGICO PARA O ALÍVIO DA DOR
}

\section{BREASTFEEDING AS A NON-PHARMACOLOGICAL METHOD FOR PAIN RELIEF}

\author{
Maria Thais de Andrade Calasans*, Júlia Martins Azevedo Maia**, Juliete Figueiredo Silva*** \\ Autora para correspondência: Júlia Martins Azevedo Maia - juliamaia2@gmail.com \\ *Enfermeira. Mestre em Enfermagem pela Universidade Federal da Bahia. Professora na Escola Bahiana de Medicina e \\ Saúde Pública. \\ **Enfermeira. Residente do Hospital Martagão Gesteira pelo Programa de Residência Multiprofissional em Saúde da \\ Criança e do Adolescente/UNIFACS. \\ ***Enfermeira. Especialização em Pediatria e Neonatologia na Escola Bahiana de Medicina e Saúde Pública.
}

\section{R E S U M O}

A dor pode ser descrita como uma experiência sensorial e emocional desagradável associada a uma lesão real ou potencial para os tecidos do corpo. A existência de escalas para a avaliação da dor, somadas às intervenções não-farmacológicas, auxilia na prevenção e no controle álgico. $\bigcirc$ presente estudo objetivou identificar a relação da amamentação como alívio da dor, comparando com outros métodos não-farmacológicos. Trata-se de uma pesquisa bibliográfica do tipo integrativa, com base em publicações no período de 2004 a 2014, disponíveis na base de dados LILACS e CINAHL, em língua portuguesa, espanhola ou inglesa, a partir dos descritores "dor", "amamentação" e "recém-nascido". A amostra final foi construída por 14 artigos que geraram duas categorias: "A dor em criança" e "O aleitamento materno como medida não farmacológica para o alívio da dor". Pesquisas realizadas nas últimas décadas não só constataram que os recém-nascidos podem sentir dor, como também, quando em estado prolongado ou repetitivo, pode trazer alterações nas funções do sistema nervoso em desenvolvimento, e, sendo assim, são suficientes para experimentar estímulos nocivos. Diversos estudos trazem medidas não farmacológicas para o controle da dor, sendo a amamentação a que obteve uma superioridade significativa quando comparada a outros métodos. Entretanto, poucos profissionais estão capacitados para identificação da dor, bem como para a realização de intervenções de alívio.

Palavras-chave: Dor; Recém-Nascido; Aleitamento Materno. 
Pain can be described as an unpleasant sensorial and emotional experience, associated to a real or potential damage to the body's tissues. The existence of scales for the evaluation of the pain, added to non-pharmacological interventions, helps in algic prevention and control. The current study had as its main goal, identifying the relation between breastfeeding and pain relief, comparing to other nonpharmacological methods. It is about an integrative bibliographic research, from publications during the period from 2004 to 2014 , available in the LILACS and CINAHL data base, in Portuguese, Spanish or English, from the descriptors "pain", "breastfeeding" and "newborn". The final sample was built by 14 articles, which generated two categories, such as: "Pain in child" and "Breast Feeding as a non-pharmacological measure to pain relief". Researches made in the last decades realized that the newborn can feel pain, as well, when in prolonged or repetitive state, can bring changes in the functions of the nervous system in development, and, therefore, are enough to try out bad stimulus. Several studies bring non-pharmacological measures for controlling pain, breastfeeding being the one that obtained a significant superiority when compared to the other methods. However, few professionals are able to identify pain, as well as achieving relief interventions.

Keywords: Pain; Newborn; Breastfeeding. 


\section{INTRODUÇÃO}

A dor pode ser descrita como uma experiência sensorial e emocional desagradável associada a uma lesão real ou potencial para os tecidos do corpo, sendo esta uma sensação subjetiva advinda de experiências dolorosas que acontecem desde o nascimento, sendo que cada indivíduo compreende seu significado'.

Durante muitos anos, a dor neonatal era desconsiderada devido a crenças sobre a imaturidade do sistema ner voso central e sua ausência de memória. Porém diversos estudos apontam que, a partir da $7^{a}$ semana de gestação, receptores sensitivos cutâneos e estruturas relacionadas à sensação dolorosa se desenvolvem. Já na $22^{a}$ semana de vida intra-uterina, o feto é capaz de sentir dor igual ou superior ao adulto, desmistificando as crenças supracitadas ${ }^{2}$. Desta forma, é necessária a conscientização dos profissionais de saúde em relação à percepção da dor nas crianças para que seja feita uma avaliação correta e, assim, atuarem de forma mais efetiva na prevenção e no controle da mesma.

Fatores ambientais e psicológicos podem exercer uma grande influência sobre as percepções de dor das crianças e serem modificados pelo uso de estratégias psicossociais, educação, apoio dos pais e intervenções congnitivo-comportamentais. O envolvimento dos pais nos cuidados com a criança junto à efetiva participação da equipe multidisciplinar só irá trazer benefícios a médio e longo prazo ${ }^{3}$.

A fim de auxiliar e atuar terapeuticamente, os profissionais de saúde utilizam instrumentos que "decodificam" a linguagem da dor, que são representadas por escalas, sendo as mais empregadas: NFCS (Neonatal Facial Coding System); NIPS (Neonatal Infant Pain Scale); CRIES (Cryng Requires $\mathrm{O} 2$ for saturation above 90\% Increased vital Signs, Expression and Sleeplessness) e PIPP (Premature Infant Pain Profile). Em sua maioria, os parâmetros utilizados para avaliação são as expressões faciais, choro, frequência respiratória, frequência cardíaca, posição das extremidades, saturação de oxigênio, estado de consciência e sono ${ }^{4}$.
Atualmente, com base na compreensão de que a dor é o quinto sinal vital, devendo ser avaliada juntamente com o pulso, a respiração, a temperatura e a pressão arterial, compete aos profissionais de enfermagem aplicar e a avaliar as escalas da dor ${ }^{3}$. Portanto, é fundamental compreender seu significado a fim de que essas intervenções para - alívio álgico façam parte do cuidado, exigindo conhecimento, habilidade e humanização para promover uma assistência qualificada levando-se em consideração sua subjetividade.

A existência dessas escalas para a avaliação da dor, somadas às intervenções não-farmacológicas, como sucção não-nutritiva, solução oral de glicose, contato pele a pele e amamentação, auxilia na prevenção e no controle álgico. $O$ contato da criança com a mãe no momento da amamentação,durante procedimentos dolorosos, promove três aspectos que auxiliam no alívio da dor: odor materno, antinociceptivo relacionado à sucção e contato pele a pele ${ }^{5}$.

presente estudo objetivou identificar a relação da amamentação com o alívio da dor, comparada com outros métodos não-farmacológicos.

\section{MÉTODOS}

Este estudo trata-se de uma pesquisa bibliográfica do tipo integrativa, buscando compreender, sob o olhar de diversos autores, a relação da amamentação como intervenção para o alívio da dor na criança, norteada pela seguinte questão: qual a relação da amamentação com o alívio da dor, comparada com outros métodos não farmacológicos?

A pesquisa bibliográfica tem por finalidade conhecer as diferentes formas de contribuição científica que se realizam sobre determinado assunto ou fenômeno(6), permitindo sua compreensão atual, a fim de esclarecer a importância de um novo estudo e servir como função integradora de conhecimentos ${ }^{7}$.

A revisão integrativa da literatura é um método de pesquisa utilizado na Prática Baseada em Evidências (PBE) que tem por objetivo reunir e 
sintetizar, de maneira sistemática e ordenada, resultados de pesquisas sobre um determinado tema ou questão. Assim, este estudo poderá contribuir para o aprofundamento do conhecimento do tema investigado e permitir a incorporação das evidências na prática clínica ${ }^{8}$.

Após a definição da pergunta norteadora (passo 1 da revisão integrativa), foi realizada uma consulta dos descritores no DeCS (Descritores em Ciências da Saúde), sendo estes "dor"; "recémnascido"; "aleitamento materno", para a validação dos mesmos. A partir desses descritores, realizouse uma busca nas bases de dados, adotando-se os seguintes critérios de inclusão: possuir texto completo em revistas indexadas em língua portuguesa, espanhola ou inglesa; textos estes publicados no período de 2004 a 2014; abordar como assunto presente o manejo da dor em crianças juntamente com o aleitamento materno; possuir como sujeito o recém-nascido e/ou lactente (passo 2).

O rastreamento dos artigos científicos foi feito em outubro de 2014 utilizando os descritores "aleitamento materno", "dor" e "recém-nascido" na base de dados LILACS (Literatura Latino Americana e do Caribe e Ciências da Saúde), na qual foram encontrados 420 artigos, e somente utilizados 06 estudos através dos critérios de inclusão. Foi realizada uma nova pesquisa no referido período utilizando os mesmos descritores na base de dados CINAHL (Cumulative Index to Nursing and Allied Health Literature), sendo rastreados 45 artigos e selecionados 08 para construção da pesquisa.

Após a coleta de dados, foram definidas informações a serem extraídas dos estudos selecionados, através da organização e sumarização das informações, em uma ficha definida para esse propósito, de maneira concisa com a proposta de formar banco de dados de fácil manejo e acesso. As informações contiveram a amostra do estudo, os objetivos, a metodologia proposta, os resultados e as principais conclusões dos estudos de interesse para esta revisão (passo 3).

\section{RESULTADOS E DISCUSSÃO}

Primeiramente, foi realizado o fichamento dos 14 artigos coletados através dos critérios de inclusão e anexados em um quadro com a finalidade de organizar e otimizar o desenvolvimento do presente estudo. Do total de artigos científicos selecionados, sete foram em forma de revisão bibliográfica (50\%); três pesquisas de campo $(21,43 \%)$; três estudos do tipo randomizado controlado $(21,43 \%)$ e um de caso-controle $(7,14 \%)$. Em relação ao ano de publicação, os anos de 2006 e 2014 apresentaram menor número de publicações sobre o tema proposto.

Quadro 1. Distribuição das pesquisas utilizadas na elaboração dos resultados - 2015.

\begin{tabular}{|c|c|c|c|c|}
\hline TíTULO & ANO & TIPO DE ESTUDO & PERIÓDICO & RELAÇÃO COM O OBJETO DE ESTUDO \\
\hline $\begin{array}{c}\text { Analgesia with } \\
\text { breastfeeding in } \\
\text { addition to skin to skin } \\
\text { contact during heel } \\
\text { prick }\end{array}$ & 2013 & $\begin{array}{c}\text { Estudo randomizado } \\
\text { controlado }\end{array}$ & $\begin{array}{c}\text { Arch Dis Child Fetal } \\
\text { Neonatal }\end{array}$ & $\begin{array}{c}\text { O aleitamento materno juntamente com o contato } \\
\text { pele a pele promove uma pontuação menor em } \\
\text { escala de dor. }\end{array}$ \\
\hline $\begin{array}{c}\text { Breastfeeding reduces } \\
\text { procedural pain in } \\
\text { infants: } \\
\begin{array}{c}\text { A review of the } \\
\text { literature }\end{array}\end{array}$ & 2014 & Revisão de literatura & Med Lab Science & A amamentação é uma forma eficaz de redução \\
da do em procedimentos dolorosos. \\
\hline $\begin{array}{c}\text { Breastfeeding on Pain } \\
\text { Relief in Full-term } \\
\text { Newborns }\end{array}$
\end{tabular}




\begin{tabular}{|c|c|c|c|c|}
\hline TíTULO & ANO & TIPO DE ESTUDO & PERIÓDICO & RELAÇÃO COM O OBJETO DE ESTUDO \\
\hline $\begin{array}{l}\text { Pain Assessment and } \\
\text { Intervention for Term } \\
\text { Newborns }\end{array}$ & 2004 & Revisão bibliográfica & $\begin{array}{l}\text { Journal of Midwifery \& } \\
\text { Women's Health }\end{array}$ & $\begin{array}{c}\text { A amamentação reduz as respostas fisiológicas da } \\
\text { criança. }\end{array}$ \\
\hline $\begin{array}{l}\text { Pain in the } \\
\text { Healthy Full-Term } \\
\text { Neonate: } \\
\text { Efficacy and } \\
\text { Safety of } \\
\text { Interventions }\end{array}$ & 2004 & Revisão bibliográfica & $\begin{array}{l}\text { Newborn and Infant } \\
\text { Nursing Reviews }\end{array}$ & $\begin{array}{l}\text { A administração oral de leite tem pouco efeito } \\
\text { frente à dor em lactentes quando comparado à } \\
\text { amamentação. }\end{array}$ \\
\hline $\begin{array}{l}\text { Procedural Pain } \\
\text { Management for } \\
\text { Neonates Using } \\
\text { Nonpharmacological } \\
\text { Strategies }\end{array}$ & 2011 & Revisão bibliográfica & $\begin{array}{l}\text { Advances in Neonatal } \\
\text { Care }\end{array}$ & $\begin{array}{l}\text { A amamentação associada ao contato pele a pele } \\
\text { está associada à redução na resposta à dor. }\end{array}$ \\
\hline $\begin{array}{l}\text { Comparison of two } \\
\text { doses of breast milk } \\
\text { and sucrose during } \\
\text { neonatal heel prick }\end{array}$ & 2010 & $\begin{array}{l}\text { Estudo prospectivo, } \\
\text { randomizado e } \\
\text { controlado }\end{array}$ & Pediatrics International & $\begin{array}{l}\text { Doses repetidas de sacarose ou leite materno não } \\
\text { foram superiores a doses individuais no alívio da } \\
\text { dor no teste do pezinho. }\end{array}$ \\
\hline $\begin{array}{l}\text { Breastfeeding as a } \\
\text { Pain Intervention When } \\
\text { Immunizing Infants }\end{array}$ & 2010 & Revisão bibliográfica & $\begin{array}{l}\text { The Journal for Nurse } \\
\text { Practitioners }\end{array}$ & $\begin{array}{l}\text { O aleitamento materno é uma intervenção natural } \\
\text { e eficaz para diminuir a percepção da dor em RN } \\
\text { durante a vacinação. }\end{array}$ \\
\hline $\begin{array}{l}\text { Pode a amentação } \\
\text { promover alívio da } \\
\text { dor aguda em recém- } \\
\text { nascidos? }\end{array}$ & 2006 & Revisão bibliográfica & $\begin{array}{l}\text { Revista Brasileira de } \\
\text { Enfermagem }\end{array}$ & $\begin{array}{l}\text { Percebe-se a eficácia da amamentação no alívio } \\
\text { da dor aguda em RNs. }\end{array}$ \\
\hline $\begin{array}{l}\text { Analgesia in newborns: } \\
\text { a case-control study of } \\
\text { the efficacy of nutritive } \\
\text { and non-nutritive } \\
\text { sucking stimuli }\end{array}$ & 2013 & Estudo caso-controle & CoDAS & $\begin{array}{l}\text { O estímulo de sucção apresenta efeito analgésico } \\
\text { no neonato se comparado a nenhum estímulo. }\end{array}$ \\
\hline $\begin{array}{l}\text { Does breastfeeding } \\
\text { reduce acute } \\
\text { procedural pain } \\
\text { in preterm infants } \\
\text { in the neonatal } \\
\text { intensive care unit? A } \\
\text { randomized clinical } \\
\text { trial }\end{array}$ & 2011 & Pesquisa de campo & PAIN & $\begin{array}{l}\text { O alívio da dor utilizando a amamentação é mais } \\
\text { eficaz em neonatos a termo. }\end{array}$ \\
\hline $\begin{array}{l}\text { Analgesic Effect of } \\
\text { Breast Milk Versus } \\
\text { Sucrose for Analgesia } \\
\text { During Heel Lance in } \\
\text { Late Preterm Infants }\end{array}$ & 2012 & $\begin{array}{l}\text { Ensaio controlado } \\
\text { randomizado }\end{array}$ & Pediatrics & $\begin{array}{c}\text { A amamentação ou leite materno suplementar } \\
\text { promove analgesia de efeito semelhante à } \\
\text { sacarose. }\end{array}$ \\
\hline $\begin{array}{c}\text { Efecto analgésico de } \\
\text { la lactancia materna } \\
\text { em la toma sanguínea } \\
\text { del talón em el recién } \\
\text { nacido }\end{array}$ & 2009 & Pesquisa de campo & Anales de Pediatría & $\begin{array}{l}\text { A amamentação é a técnica mais eficaz para } \\
\text { analgesia da dor aguda à moderada. }\end{array}$ \\
\hline $\begin{array}{l}\text { Breastfeeding } \\
\text { or breastmilk for } \\
\text { procedural pain in } \\
\text { neonates }\end{array}$ & 2012 & Revisão bibliográfica & The Cochrane Library & $\begin{array}{c}\text { O aleitamento materno proporciona alívio da dor } \\
\text { em recém-nascidos. }\end{array}$ \\
\hline
\end{tabular}

Fonte: Elaborado pelas próprias autoras, 2015.

A partir da leitura dos artigos selecionados, definiram-se duas categorias, para uma melhor estruturação e compreensão do tema abordado, sendo essas: "A dor em crianças" e "O aleitamento materno como medida não farmacológica para o alívio da dor".

\section{A DOR EM CRIANÇAS}

No período intra-uterino, a criança desenvolve estruturas anatômicas, fisiológicas e neuroquímicas responsáveis pela transmissão da dor. Os diversos estudos realizados nas últimas décadas não só 
constataram que os recém-nascidos podem sentir dor, como também, quando em estado prolongado ou repetitivo,pode trazer alterações nas funções do sistema nervoso em desenvolvimento, e, sendo assim, são suficientes para experimentar estímulos nocivos $^{9-11}$. É evidenciado pela literatura clássica que, devido à organização dos sistemas nervosos central e periférico, a sensação de dor tende a reduzir ainda mais o seu limiar quando o recémnascido é estimulado de forma prolongada ou repetitiva $^{12}$.

A resposta à dor neste público envolve causas fisiológicas e instabilidade comportamental através do aumento na produção de cortisol, provocando um crescimento metabólico e assim uma maior suscetibilidade a complicações ${ }^{13,14}$. Com $\circ$ intuito de impedir ou limitar a duração da experiência dolorosa, o organismo desenvolve ações como: aumento da frequência cardíaca, variabilidade na frequência respiratória, modificações comportamentais e consequente aumento do gasto energético'. Contudo, é necessário compreender a individualidade de cada um, que pode apresentar comportamentos subjetivos, como o ato de não chorar.

A criança tem a capacidade de lembrar-se da sensação dolorosa a partir do momento que sofre o estímulo ${ }^{1,14}$, porém este deve ser tratado de forma peculiar já que nem sempre é possível referir esta sensação, atestando a necessidade de promover um olhar mais cauteloso. O tratamento da dor na criança torna-se de extrema importância já sendo conhecida como o quinto sinal vital pela Sociedade Americana de Dor e pela Agência Americana de Pesquisa e Qualidade em Saúde Pública, e não ter um olhar cuidadoso para isso pode ser considerado um ato de negligência por parte do profissional de saúde ${ }^{10}$.

De acordo com a Comissão Conjunta de Acreditação de Organizações de Saúde, o indivíduo deve ter uma avaliação inicial e um plano de manejo da dor, para isso é necessário que este plano esteja incluso na sistematização da assistência de enfermagem ${ }^{15}$. Por conseguinte, o Ministério da Saúde evidencia que a utilização de escalas, que tem como objetivo decifrar o nível de dor na criança, deve ser repetida regularmente e de forma sistematizada para promover uma melhor qualidade na assistência à saúde ${ }^{16}$.

Além das escalas utilizadas no manejo da dor, intervenções não farmacológicas estão disponíveis para serem utilizadas de forma isolada ou combinada com o método farmacológico ${ }^{15}$. Porém, é observado que poucos profissionais fazem uso das mesmas, muitas vezes por dificuldade na identificação e/ou sensibilização da dor como também por desconhecimento.

Dentre os métodos não farmacológicos utilizados nas pesquisas, a amamentação foi a mais aplicada por ser o foco do estudo, no entanto, não é a opção mais empregada pelos profissionais na prática ${ }^{1}$. Estudos apresentam a eficácia deste método quando usado como uma distração para dor infantil ${ }^{13}$, traduzindo efeitos benéficos tanto para a criança quanto para - processo de trabalho da equipe de saúde, o que reflete a necessidade de conscientização dos profissionais que trabalham com o público neonatal e pediátrico.

Para que a assistência de enfermagem seja efetiva no controle da dor, gestores dos serviços de saúde, juntamente com o setor de educação permanente, poderiam elaborar protocolos de atendimento e oferecer capacitação aos profissionais na utilização das escalas e medidas não farmacológicas para o alívio da dor. Desta forma, o profissional exerceria uma assistência qualificada no sentido de incluir a dor como um sinal vital que necessita de intervenções contínuas dentro do processo de enfermagem.

\section{O ALEITAMENTO MATERNO COMO MEDIDA NÃO FARMACOLÓGICA PARA O ALÍVIO DA DOR}

O leite materno possui a vantagem de ser um método natural e de baixo custo, além de proporcionar o contato pele a pele entre mãe e bebê ${ }^{13}$. Estudos apontam a eficácia de alguns componentes do leite materno no alívio da dor do recém-nascido, já que ele contém o triptofano, um precursor da melatonina que aumenta a beta endorfina e pode auxiliar no processo álgico ${ }^{17}$. Outro componente é o odor do leite materno que pode contribuir de forma significativa para o alívio da dor, visto que em dois estudos com crianças a termo, houve uma melhor 
resposta ao choro e aumento da sucção quando comparado a crianças expostas a nenhum odor ${ }^{18,19}$.

Há uma relação entre odor, sucção, contato e sabor do leite materno, e para a qual alguns autores trazem como medidas não-farmacológicas para diminuição da dor em recém-nascidos, pois influenciam diretamente na percepção e amadurecimento dos mesmos $^{11,19}$. A associação da amamentação com o contato pele a pele traduz formas positivas sobre a analgesia, além de fornecer um método natural e disponível para a maioria das mães, pois o ato de mamar reduz o gasto energético através da redução dos parâmetros fisiológicos, como choro e a atividade motora ${ }^{20}$. A presença materna é um fator contribuinte nesse processo, envolvendo participação ativa e emocional em um período que causa desconforto para ambos, e para o qual é necessário o reconhecimento do profissional acerca da importância da amamentação relacionada à analgesia.

Uma pesquisa com estímulos multissensoriais relacionadas ao processo da dor revela que bebês expostos ao odor do leite materno expressam menos agitação motora durante e após a punção de calcanhar $^{18}$. O ato de amamentar traduz na mãe um contato íntimo observado através do efeito calmante do odor do leite, apresentando níveis de cortisol diminuídos à criança o que permite sua participação ativa no controle da dor e medidas de conforto ${ }^{17}$. Os estudos fornecem evidências de que os bebês têm capacidade de recordar odores familiares, sendo o materno capaz de mediar a resposta da dor.

Ao analisar recém-nascidos a termo, utilizando a escala NFCS durante o teste do pezinho, foi dado leite materno cinco minutos antes do procedimento, resultando em menores escores na escala e, consequentemente, efeitos benéficos. 0 ritmo cardíaco, o estado sono-vigília e o estímulo da sucção apresentaram melhores resultados comparados a outros grupos, mostrando a eficácia da amamentação como analgésico em momentos de dor rápida a moderada ${ }^{5,9}$.

Porém, ao verificar uma pesquisa utilizando o mesmo método e procedimento, não houve efeito analgésico significativo ao introduzir dose única ou dose dupla de leite materno, o que traduz poucos estudos sobre o efeito antinociceptivo do mesmo ${ }^{21}$. Tal fato pode estar relacionado à introdução do leite materno somente antes do procedimento doloroso, contrapondo outros estudos que obtiverem resultados positivos através dessa intervenção antes, durante e após o procedimento. Contudo, destacase a importância de realizar novas pesquisas para aprofundar esse assunto tão presente na área da saúde.

Ao ser avaliado um estudo caso controle e outro de pesquisa de campo, durante a punção venosa e de calcanhar em neonatos a termos, foi aplicada a escala NIPS, resultando em menores pontuações nos grupos de sucção nutritiva (aleitamento) comparado a grupos de sacarose, de sucção não-nutritiva ou que não receberam nada1,15,20. A oferta do leite se deu de dois a cinco minutos antes, durante $e$ após $\circ$ procedimento $\circ$ que refletiu a importância do preparo da criança submetida à dor, a fim de minimizá-la.

Em contrapartida, outro artigo traz que o colostro associado à chupeta (sucção nutritiva) não promove analgesia, de acordo com os índices comportamentais (choro e mímica facial). Entretanto, o mesmo foi tão efetivo quanto a sacarose no que diz respeito à prevenção da taquicardia durante $e$ após a punção ${ }^{19}$.

Outro aspecto mencionado em uma revisão bibliográfica, ainda tratando-se de punção de calcanhar, mostra que os recém-nascidos amamentados obtiveram valores menos significantes na escala NFCS quando comparados ao grupo de crianças no berço enroladas no cobertor, como também menores valores na frequência cardíaca ${ }^{19}$.

Em um dos experimentos analisados foi evidenciado que os grupos de sucção nutritiva e não-nutritiva não obtiverem diferença estatisticamente significante a estímulos nocivos ao realizar punção venosa, supondo a eficácia do estímulo de sucçãol. Esta análise também pode ser observada em outro artigo no qual o ato de amamentar, ou seja, realizar a sucção nutritiva, foi superior ao nível de analgesia comparado à oferta de leite materno através de seringa ${ }^{10}$.

Na população pré-termo, aplicou-se a escala de PIPP, que envolve uma avaliação multidimensional da 
dor, constatando mesma resposta ao alívio álgico na relação amamentação e administração de glicose durante procedimentos pequenos, como punção de calcanhar e aplicação de vacinas ${ }^{11,14}$. Tal fato pode acontecer devido à imaturidade dos prematuros em realizar a sucção, interrompendo facilmente o processo da amamentação. Entretanto, a escala de PIPP no mesmo tipo de população, mas em outro estudo, apresentou menores escores no grupo da amamentação quando relacionado ao grupo de glicose oral, no que diz respeito à freqüência cardíaca e tempo de choro ${ }^{22}$. A única pesquisa que não reduziu os índices de dor comportamental e fisiológica foi em prematuros durante a coleta sanguínea ${ }^{13}$, sendo esses os pacientes que possuem maior tempo de internação e, consequentemente, submetidos a mais procedimentos dolorosos e merecedores de pesquisas relevantes.

Embora nenhum efeito global relacionado a resposta à dor foi encontrado, as crianças com comportamento mais maduro obtiveram índices mais baixos ${ }^{13}$, refletindo uma possível relação da idade gestacional com a dor. Portanto, o tempo de desenvolvimento intra-uterino poderá refletir diretamente na resposta fisiológica do recémnascido.

O efeito da sucção na criança é visto como coadjuvante no processo estressante da dor. A utilização de glicose $30 \%$ com o estímulo da sucção (chupeta) foi tão eficaz como a amamentação em um estudo de campo. Já a suç̧ão, juntamente com o contato pele a pele, traduz uma melhor resposta à analgesia quando utilizados individualmente ${ }^{14}$.

Ao comparar os efeitos fisiológicos, a sacarose obteve um menor tempo de choro relacionado à amamentação além de redução na resposta à dor em neonatos. Entretanto, um determinado estudo identificou riscos na administração da sacarose, evidenciado por asfixia e dessaturação ${ }^{10,19,21}$. Foi verificado que, ao utilizar o mesmo método em recém-nascidos prematuros, não houve evidências de que a sacarose seja superior ao leite materno, quando usada em casos de dificuldade no processo de amamentação' ${ }^{11}$.

Em uma pesquisa realizada em alojamento conjunto observou-se que existe uma deficiência por parte dos profissionais no que diz respeito aos métodos não-farmacológicos de alívio da dor nos recémnascidos'. Essa observância nos fez refletir sobre a causa que pode estar levando esses profissionais a chegarem ao sistema de saúde sem um conhecimento que é basal e promove o que há de elementar na assistência: o conforto dos pacientes.

\section{CONSIDERAÇÕES FINAIS}

A dor é sensitiva na criança desde o período intra-uterino, sendo considerada em grau maior e/ou igual comparada à idade adulta, logo, fica evidente a necessidade do conhecimento de formas para $\circ$ alívio álgico nesta fase da vida. Diversos estudos trazem medidas não farmacológicas para - controle da dor, sendo a amamentação a que obteve uma superioridade significativa quando comparada a outros métodos.

O contato pele a pele, junto com o odor do leite materno e a sucção, mostraram-se de grande magnitude nos estudos realizados. Além dos benefícios do leite, o contato materno estabelece um vínculo afetivo e psicológico para a criança que se traduz em momentos únicos que trazem benefícios nos processos estressores.

O método não-farmacológico que mais se igualou aos benefícios da amamentação no alívio da dor foi a utilização da sacarose. Foram evidenciados menores parâmetros fisiológicos, porém há pouco embasamento nas pesquisas realizadas e falta de interesse em realizar novos estudos.

Neste estudo foi possível observar que existem poucos artigos que tratam da amamentação como intervenção para o alívio da dor no recémnascido e/ou lactente em nosso país, o que traduz a dificuldade de encontrar artigos de origem brasileira. Além disso, verificamos que, apesar de ser um tema muito importante para quem lida com o público infantil, poucos profissionais estão capacitados para identificação da dor, bem como para a realização de intervenções de alívio.

Os estudos analisados trazem diversas formas de redução da dor, mas pouco se fala sobre a sua 
combinação eficácia quando usadas em conjunto. $\bigcirc$ efeito da sucção mostrou-se como coadjuvante neste processo estressor da dor, o que revela um efeito benéfico do leite materno para aliviar a dor.

\section{REFERÊNCIAS}

1. Lima AH, Hermont AP, Friche AAL. Analgesia in newborns: a case-control study of the efficacy of nutritive and non-nutritive sucking stimuli. CoDAS. 2013;25(4):365-368. doi: 10.1590/S231717822013005000002

2. Souza $A B G$. Enfermagem em neonatologia: temas relevantes. São Paulo: Martinari;2010.

3. Hockenberry MJ. Wong: Fundamentos de enfermagem pediátrica. $9^{a}$ Ed. Rio de Janeiro: Elsevier; 2014.

4. Crescêncio EP, Zanelato $S$, Leventhal LC. Avaliação e alívio da dor no recém-nascido.Rev. Eletr. Enf. 2009;1 1 (1):64-9

5. Leite $A M$, Linhares $M B M$, Lander J, Castral TC, Santos CB, Scochi CGS. Effects of breastfeeding on pain relief in full-term newborns. Clin J Pain. 2009;25:827-832. doi: 10.1097// AJP.0b013e3181b51191

6. Oliveira SL. Tratado de Metodologia Científica: projetos de pesquisa, TGI, TCC, monografias, dissertações e teses. São Paulo: Pioneira Thomson Learning; 2001.

7. Polit DF, Beck CT, Hungler BP. Fundamentos de pesquisa em enfermagem: métodos, avaliação e utilização. $5^{\mathrm{a}}$ ed. Porto Alegre: Artmed; 2004.

8. Mendes KDS, Silveira RCCP, Galvão CM. Revisão integrativa: método de pesquisa para a incorporação de evidências na saúde e na enfermagem. Texto contexto - enferm. 2008;17(4):758-764. doi: 10.1590/S010407072008000400018

9. Iturriagaa AGS, Unceta-Barrenecheaa KA,
Zarateb IS, Olaecheab AZ. Efecto analgésico de la lactancia materna en la toma sanguínea del talón en el recién nacido. AnPediatr (Barc). 2009;71 (4):310-3. doi: 10.1016/i. anpedi.2009.06.023

10. Henry PR, Haubold K, Dobrzykowsk TM. Pain in the Healthy Full-Term Neonate: Efficacy and Safety of Interventions. Newborn and Infant Nursing Reviews. 2004;4(2);106-113. doi: 10.1053/i. nainr.2004.03.002

11. Simonse E, Mulder PGH, Van BeekRHT. Analgesic Effect of Breast Milk Versus Sucrose for Analgesia During Heel Lance in Late Preterm Infants. Pediatrics. 201 2; 129(4):657-663. doi: $10.1542 /$ peds. $2011-2173$

12. Silva L. Diagnóstico em pediatria: Guanabara Koogan; 2009.

\section{Holsti L, Oberlander TF, Brant R. Does} breastfeeding reduce acute procedural pain in preterm infants in the neonatal intensive care unit? A randomized clinical Trial. Pain. $2011 ; 152(11$ ):2575-81. doi: 10.1016/i. pain.2011.07.022

14. Tansky C, Lindberg CE. Breastfeeding as a Pain Intervention When Immunizing Infants. Journal for Nurse Practitioners. 2010;6(4):287-95. doi: 10.1016/i.nurpra.2009.09.014

15. Clifford PA, Stringer $M$, Christensen $H$, Mountain D. Pain Assessment and Intervention for Term Newborns. J MidwiferyWomens Health. 2004;49(6):514-9. doi: 10.1016/i. jmwh.2004.07.008

16. Brasil. Ministério da Saúde. Secretaria de Atenção à Saúde. Departamento de Ações Programáticas Estratégicas. Atenção à saúde do recém-nascido: guia para os profissionais de saúde. $2^{a}$ ed. Brasília. DF. 2014.

\section{Appleyard LR. Breastfeeding reduces} procedural pain infants: A review of the literature. NZ J Med Lab Science. 2014;68:88-89.

18. Campbell-Yeo M, Fernandes A, Johnston C. Procedural Pain Management for Neonates Using 
Non pharmacological Strategies. Advances in Neonatal Care. $2011 ; 11(5): 312-18$.

19. Leite AM, Castarl TC, Scochi CGS. Pode a amamentação promover alívio da dor aguda em recém-nascidos? Rev. bras. enferm. 2006; 59(4):538-42. doi: 10.1590/S003471672006000400012

20. Gabriel MAM, Mendonza BRY, Figuroa LJ, Medina $\mathrm{V}$, Fernández BI, Rodríguez MV etal. Analgesia with breastfeeding in addition to skin-toskin contact during heel prick. Arch Dis Child Fetal Neonatal. 2013;98(6):499-503. doi: 10.1136/ archdischild-2012-302921

21. Akman TOI, Cebeli D, Bilgen H, Ozek E. Comparison of two doses of breast milk and sucrose during neonatal heel prick. Pediatrics International. 2010; 52(2):175-9. doi: $10.1111 /$ i.1442-200X.2009.02921.x

22. Shah PS, Herbozo C, Aliwas LL. Breastfeeding or breast milk for procedural pain in neonates. Cochrane Database Syst Rev. 201 2; 12:CD004950. doi: 10.1002/14651858.CD004950.pub3 